\title{
Spatio-temporal organization of vehicles in a cellular automata model of traffic with 'slow-to-start' rule
}

\author{
Debashish Chowdhury*, ${ }^{*}$, Ludger Santen ${ }^{\dagger}$, Andreas Schadschneider ${ }^{\dagger}$, \\ Shishir Sinha*円, and Abhay Pasupathy*
}

${ }^{*}$ Physics Department, Indian Institute of Technology, Kanpur 208016, India

$\dagger$ Institute for Theoretical Physics, University of Cologne, D-50923 Köln, Germany

\begin{abstract}
The spatio-temporal organizations of vehicular traffic in cellular-automata models with "slow-to-start" rules are qualitatively different from those in the NagelSchreckenberg (NaSch) model of highway traffic. Here we study the effects of such a slow-to-start rule, introduced by Benjamin, Johnson and Hui (BJH), on the the distributions of the distance-headways, time-headways, jam sizes and sizes of the gaps between successive jams by a combination of approximate analytical calculations and extensive computer simulations. We compare these results for the BJH model with the corresponding results for the NaSch model and interpret the qualitative differences in the nature of the spatio-temporal organizations of traffic in these two models in terms of a phase separation of the traffic caused by the slow-to-start rule in the BJH model.
\end{abstract}

PACS: 05.40.+j; 05.60.+w; 89.40.+k.

\footnotetext{
${ }^{1}$ Present address: Department of Physics, Harvard University, Cambridge, MA 02138, U.S.A.

${ }^{2}$ Present address: LASSP, Cornell University, Ithaca, NY 14853, U.S.A.
} 


\section{Introduction}

The continuum theories of the flow of vehicular traffic [1 13$]$ are analogous to the hydrodynamic description of fluid flow. The kinetic theories of vehicular traffic [146] are extensions of the kinetic theory of gases whereas the "car-following models" 17 10] and the recent particle-hopping models [11 29] are analogues of the models of driven system of interacting particles [30]. Therefore, the theoretical techniques of fluid dynamics and non-equilibrium statistical mechanics have turned out to be powerful tools in the study of a wide variety of problems in the science and engineering of vehicular traffic [31 33]. One of the most popular "particle-hopping models" of vehicular traffic is the Nagel-Schreckenberg (NaSch) model 11], which may be regarded as a stochastic cellular automaton (CA) [34], where the timeevolution of the vehicles is formulated in terms of a set of "rules". Several extensions of these rules have been suggested in recent years (see, e.g., [21 29] ) to make the model more realistic; in this paper we study some of the statistical properties of one such extension 25.

The flux versus density of the vehicles is known as the fundamental diagram [31]. The distance-headway $(\mathbf{D H})$ is defined as the distance from a selected point on the lead vehicle $(\mathbf{L V})$ to the same point on the following vehicle $(\mathbf{F V})$; usually the front edges or bumpers are selected [31]. The time-headway (TH) is defined as the time interval between the departures (or arrivals) of two successive vehicles recorded by a detector placed at a fixed position on the highway [31]. The fundamental diagram, the $\mathrm{DH}$ and $\mathrm{TH}$ distributions, the distributions of the sizes of the traffic jams as well as the distribution of the gaps between successive jams in the NaSch model on idealized single-lane highways have been calculated 11 20. Very recently, the update "rules" of the NaSch model have been extended by adding a step where the so-called "slow-to-start" rules (which will be explained in the next section) 2528 are implemented. The fundamental diagram of some of these CA models with "slow-to-start" rules have been investigated [28]. The main aim of this paper is to calculate, by a combination of analytical and numerical methods, the distributions of $\mathrm{DH}, \mathrm{TH}$, jam sizes and distances between jams in the CA model with the "slowto-start" rule suggested by Benjamin, Johnson and Hui (BJH) 25] to study the effects of the slow-to-start rule on the spatio-temporal organization of traffic. We interpret the novel features of these distributions in terms of a phase separation of the traffic in the BJH model.

The NaSch model [11] and the BJH model [25], which was formulated by BJH by incorporating one specific type of "slow-to-start" rule in the updating scheme of the NaSch model, are stated in section 2 for the sake of completeness. The theoretical techniques, which will be used in our analytical calculations, and the numerical methods, which will be followed in our computer simulations, are also explained briefly in the same section. The probability distributions, namely the distributions of $\mathrm{DH}, \mathrm{TH}$, jam sizes and gaps between jams, with which we are mainly concerned in this paper, are defined in section 2. Our results for the DH distributions are presented in section 3. Our analytical as well as numerical results for the distributions of jam sizes and jam gaps are presented in sections 4 and 5, respectively, while those for the $\mathrm{TH}$ distributions are given in section 6 . We make a quantitative estimation of the regime of validity of our analytical expressions by computing an appropriate correlation function in section 7. A summary of our results and conclusions drawn from these are given in section 8. Three appendices contain details of our analytical calculations. 


\section{Models and theoretical techniques}

In the particle-hopping models a lane is represented by a one-dimensional lattice of $L$ sites. Each of the lattice sites can be either empty or occupied by at most one "vehicle". If periodic boundary condition is imposed, the density $c$ of the vehicles is $N / L$ where $N(\leq L)$ is the total number of vehicles. Throughout this paper we shall follow the convention that the vehicles move from left to right (i.e., along the positive $X$-axis), so that in a given configuration $(a, b)$, of a pair of sites, $b$ refers to the LV and $a$ refers to the FV.

\section{$2.1 \quad$ The models}

\subsubsection{The NaSch model}

For the sake of completeness we briefly recall the definition of the NaSch model 11]. In this model, the speed $V$ of each vehicle can take one of the $V_{\max }+1$ allowed integer values $V=0,1, \ldots, V_{\max }$. Suppose, $X_{n}$ and $V_{n}$ denote the position and speed, respectively, of the $n$-th vehicle. Then, $d_{n}=X_{n+1}-X_{n}$, is the gap in between the $n$-th vehicle and the vehicle in front of it at time $t$. At each discrete time step $t \rightarrow t+1$, the arrangement of $N$ vehicles is updated in parallel according to the following "rules":

Step 1: Acceleration. $V_{n} \rightarrow \min \left(V_{n}+1, V_{\max }\right)$.

Step 2: Deceleration (due to other vehicles). $V_{n} \rightarrow \min \left(V_{n}, d_{n}-1\right)$.

Step 3: Randomization. $V_{n} \rightarrow \max \left(V_{n}-1,0\right)$ with probability $p$.

Step 4: Vehicle movement. $X_{n} \rightarrow X_{n}+V_{n}$.

Non-vanishing braking probability $p$ is essential for a realistic modeling of traffic flow [13] and, therefore, the NaSch model may be regarded as stochastic cellular automata [33].

\subsubsection{The BJH model}

To our knowledge, so far three different versions of the "slow-to-start" rule have been formulated [25 28] ; in this paper we shall consider only the slow-to-start rule introduced by Benjamin et al. (BJH) 25. BJH modified the updating rules of the NaSch model by introducing an extra step where their "slow-to-start" rule is implemented. According this "slow-to-start" rule, the vehicles which had to brake due to the next vehicle ahead will move on the next opportunity only with probability $1-p_{s}$. The steps of the update rules can be stated as follows:

Step 1: Acceleration. $V_{n} \rightarrow \min \left(V_{n}+1, V_{\max }\right)$.

Step 2: Slow-to-start rule: If flag $=1$, then $V_{n} \rightarrow 0$ with probability $p_{s}$.

Step 3: Blockage (due to other vehicles). $V_{n} \rightarrow \min \left(V_{n}, d_{n}-1\right)$ and, then,

flag $=1$ if $V_{n}=0$, else $f l a g=0$.

Step 4: Randomization. $V_{n} \rightarrow \max \left(V_{n}-1,0\right)$ with probability $p$.

Step 5: Vehicle movement. $X_{n} \rightarrow X_{n}+V_{n}$.

Here flag is a label distinguishing vehicles which have to obey the slow-to-start rule $($ flag $=1)$ from those which do not have to $($ flag $=0)$.

For $p_{s}=0$ the above rules reduce to those of the NaSch model. Note that, in both these models, if $V_{\max }=1$, a vehicle can come to an instantaneous halt, even at vanishingly low densities, because of random braking. In contrast, for all $V_{\max }>1$, vehicles do not stop spontaneously at sufficiently low densities for which interactions among the vehicles is negligibly small. Therefore, effectively free flow of traffic takes place when the density of vehicles is sufficiently low whereas high density leads to congestion and traffic jams. The density, $c_{o}$, corresponding to the maximum flux is usually called the optimum density. At sufficiently high densities, 
the BJH model with $V_{\max }>1$ is known to exhibit phase separation where one of the two phases consists of a macroscopic jam while the other corresponds to free flow of traffic. Moreover, the particle-hole symmetry of the NaSch model for $V_{\max }=1$ is lost in the corresponding BJH model because of the slow-to-start rule [28].

\subsection{Theoretical techniques}

We compute the DH distribution in the BJH model with $V_{\max }=1$ using some analytical results derived earlier [28] within the framework of a car-oriented meanfield (COMF) theory [14]. Our analytical calculations of the distributions of jam sizes in the BJH model with $V_{\max }=1$ are also carried out within the framework of the same COMF theory. We derive analytical expressions for the distributions of the gaps between successive jams in the BJH model for $V_{\max }=1$ using a 2-cluster site-oriented mean-field (SOMF) theory which leads to the exact results for the NaSch model with $V_{\max }=1$ in the limit $p_{s} \rightarrow 0$, although the results for the BJH model $\left(p_{s} \neq 0\right)$ are not exact. We also present an alternative derivation of the distributions of the gaps between successive jams in terms of a hybrid approach which will be explained in section 5. Finally, we derive the analytical expression for the TH distributions in the BJH model with $V_{\max }=1$ following an extension of the approach used earlier [19] for the corresponding calculation for NaSch model.

In our computer simulations we begin with a random initial configuration and let a system of $L=1000$ sites evolve for $10^{4}$ steps which is long enough to ensure that the system reaches the steady state; then we let the system evolve for $10^{5}$ further steps during which we compute the properties of our interest. Then the data are averaged over 100 samples (i.e., 100 initial configurations) for each of the sets of values of the parameters. We have analyzed data for a wide range of values of the parameters $p$ and $p_{s}$. However, we present here data mainly for the regime $p \ll p_{s}$ where effects of the slow-to-start rule are the strongest.

\subsection{The distributions of our interest}

In this section we define the distributions of our interest. The number of empty lattice sites in front of a vehicle is taken to be a measure of the corresponding $\mathrm{DH}$. Suppose $\mathcal{P}_{d h}(k)$ denotes the probability of a DH $k$; more precisely, $\mathcal{P}_{d h}(k)$ is the conditional probability of finding a string of $k$ empty sites in front of a site which is given to be occupied by a vehicle. Note that $\mathcal{P}_{d h}(0)$ is also the total fraction of vehicles which are simultaneously in the jammed state.

A jam of length $k$ is defined as a string of $k$ successive stopped vehicles, i.e. we are considering only compact jams. Similarly, when there are $k$ lattice sites between two successive jams, each occupied by a moving vehicle or is vacant then we say that there is a gap of length $k$ between the two successive jams; we are interested in the distribution $\mathcal{P}_{j g}(k)$.

We define the $\mathrm{TH}$ as follows [18]. A detector is placed at one of the lattice sites. In order to measure the $\mathrm{TH}$ between a given pair of vehicles the detector is set to $t=0$ instantaneously when the LV of the pair leaves the detector site. From then onwards the detector counts the number of discrete time steps up to $t=\tau$ when the FV leaves the same detector site; $\tau$ is the TH between the pair of vehicles under consideration. As soon as the FV leaves the detector site the detector instantaneously resets again to $t=0$ in order to measure the TH between this vehicle and the vehicle following it. We denote the TH distribution by the symbol $\mathcal{P}_{t h}(\tau)$. 


\section{The DH distribution}

Suppose, $\mathcal{P}(k)$ denotes the probability to find exactly $k$ empty cells in front of a vehicle. Only vehicles which have exactly one empty cell in front might be affected by the slow-to-start rule [28]. We denote the density of these vehicles by $\tilde{\mathcal{P}}(1)$. The normalization requires

$$
\sum_{k \geq 0} \mathcal{P}(k)+\tilde{\mathcal{P}}(1)=1 .
$$

Note that $\mathcal{P}_{d h}(1)=\mathcal{P}(1)+\tilde{\mathcal{P}}(1)$, and $\mathcal{P}_{d h}(k)=\mathcal{P}(k)$ for all $k \neq 1$. The symbol $g(t)$ $(\bar{g}(t))$ is the probability that the vehicle moves (does not move) in the next time step; therefore,

$$
g=q \sum_{k \geq 1} \mathcal{P}(k)+q q_{s} \tilde{\mathcal{P}}(1)
$$

where $q=1-p$ and $q_{s}=1-p_{s}$. Making the ansatz

$$
\mathcal{P}(k)=\mathcal{N} z^{k} \quad(k \geq 2)
$$

one gets the following results [28]:

$$
\begin{gathered}
\mathcal{P}(1)=\frac{z \mathcal{P}(0)}{p}-q_{s} \tilde{\mathcal{P}}(1) \\
\tilde{\mathcal{P}}(1)=\frac{q \mathcal{P}(0)(1-\mathcal{P}(0))}{1+p_{s} q \mathcal{P}(0)}
\end{gathered}
$$

where

$$
\begin{gathered}
\mathcal{N}=\frac{1-z}{z^{2}} \cdot[1-\mathcal{P}(0)-\mathcal{P}(1)-\tilde{\mathcal{P}}(1)], \\
z=\frac{p g}{q(1-g)}, \\
g=\frac{q(1-\mathcal{P}(0))}{1+p_{s} q \mathcal{P}(0)}
\end{gathered}
$$

and $\mathcal{P}(0)$ is given as the root in the interval $(0,1)$ of the cubic equation

$$
\begin{aligned}
c p_{s}^{2} q^{2} \mathcal{P}(0)^{3} & +q\left[q p_{s}^{2}(1-2 c)+p_{s}(1+c)+c\right] \mathcal{P}(0)^{2} \\
& +\left[q p_{s}(1-3 c)-2 q c+1\right] \mathcal{P}(0)-p c=0
\end{aligned}
$$

In the special limit $p_{s} \rightarrow 0$,

$$
\begin{gathered}
\tilde{\mathcal{P}}(1) \rightarrow \frac{q y}{c}\left(1-\frac{y}{c}\right), \\
g \rightarrow \frac{q y}{c},
\end{gathered}
$$

and

$$
z \rightarrow \frac{(p y / c)}{1-(q y / c)}=1-\frac{y}{1-c}
$$


where

$$
y=\frac{1}{2 q}[1-\sqrt{1-4 q c(1-c)}]
$$

is the solution of the quadratic equation

$$
q y^{2}-y+c(1-c)=0 .
$$

Moreover, in the special case $p_{s}=0$, the cubic equation (9) for $\mathcal{P}_{d h}(0)$ reduces to the quadratic equation

$$
q c \mathcal{P}_{d h}(0)^{2}+(1-2 q c) \mathcal{P}_{d h}(0)-p c=0,
$$

whose physically relevant solution

$$
\mathcal{P}_{d h}(0)=\frac{(2 q c-1)+\sqrt{(2 q c-1)^{2}+4 p q c^{2}}}{2 q c}=1-\frac{y}{c}
$$

is identical with the corresponding exact result [14, 17] for the NaSch model. Thus, in the limit $p_{s} \rightarrow 0$,

$$
\mathcal{P}_{d h}(1)=\mathcal{P}(1)+\tilde{\mathcal{P}}(1) \rightarrow \frac{y^{2}}{c(1-c)}
$$

and

$$
\mathcal{P}_{d h}(k) \rightarrow \frac{y^{2}}{c(1-c)} \cdot\left[1-\frac{y}{1-c}\right]^{k-1} \quad \text { for } k \geq 1,
$$

which are the corresponding known results for the NaSch model [14, 17].

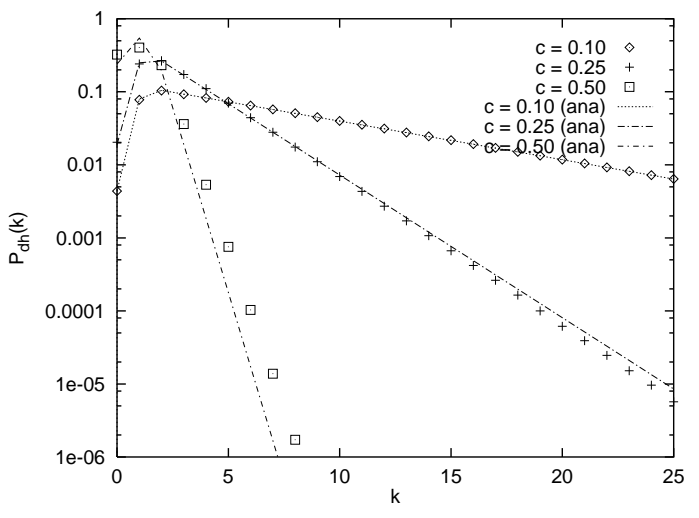

Figure 1: The DH distributions in the BJH model with $V_{\max }=1$ are shown on a semi-log plot for three different values of the density of vehicles $c$. The lines are obtained from the approximate analytical expressions for $\mathcal{P}_{d h}(k)$ while the discrete data points have been obtained from computer simulation. The common parameters are $p=0.05, p_{s}=0.50$.

For $V_{\max }=1$, and for given $c$, we have solved the cubic equation (9) numerically for $\mathcal{P}(0)$ and, hence, computed $g, z, \tilde{\mathcal{P}}(1), \mathcal{P}(1), \mathcal{N}, \mathcal{P}(k)$ for all $k \geq 2$. Then, we calculated the distance-headway $\mathcal{P}_{d h}(k)$ for all $k$ in the BJH model corresponding to the density $c$. A comparison of these results with those obtained from computer simulation for the same set of parameters (fig. I) establishes that for low densities, e.g. $c=0.10$, and $c=0.25$, the approximate analytical expressions for $\mathcal{P}_{d h}(k)$ derived in the COMF approximation, are in good agreement with the simulation data. Larger deviations of the analytical results are observable for $c=0.5$. 


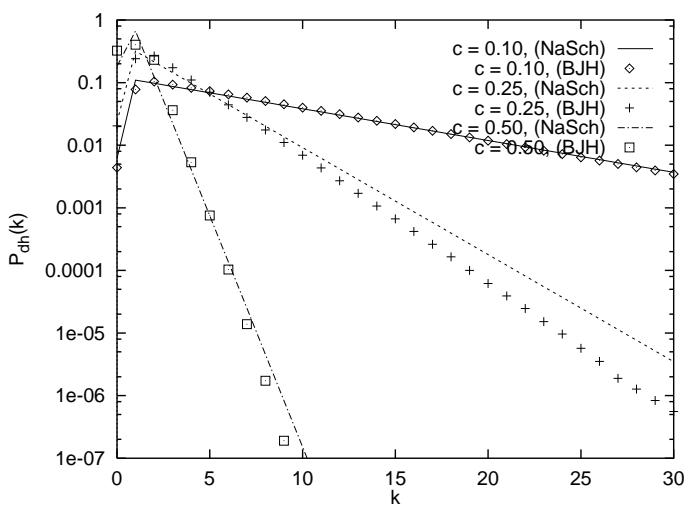

Figure 2: The DH distributions in the BJH model and the NaSch model, both with $V_{\max }=1$ and $p=0.05$, are shown on a semi-log plot for three different values of the density of vehicles $c$. The lines are obtained from the exact analytical expressions for $\mathcal{P}_{d h}(k)$ in the NaSch model while the discrete data points have been obtained from computer simulation of the BJH model with $p_{s}=0.50$.

We have made a comparison of the $\mathrm{DH}$ distributions in the NaSch model and the BJH model, both with $V_{\max }=1$ and with $p=0.05$, in fig. 2. At the low density of $c=0.1$ there is practically no difference in the $\mathrm{DH}$ distributions in these two models when $p=0.05$. However, at the moderately high density of $c=0.25$, large $\mathrm{DH}$ are slightly less probable in the BJH than in the NaSch model. Surprisingly, the difference in the DH distributions in these two models is less significant at $c=0.5$ than at $c=0.25$. This, we believe, is a consequence of the fact that at a high density of $c=0.5$ the $\mathrm{DH}$ distribution is decided mainly by the blockage of one vehicle by another, rather than the slow-to-start rule. Some typical DH distributions in the

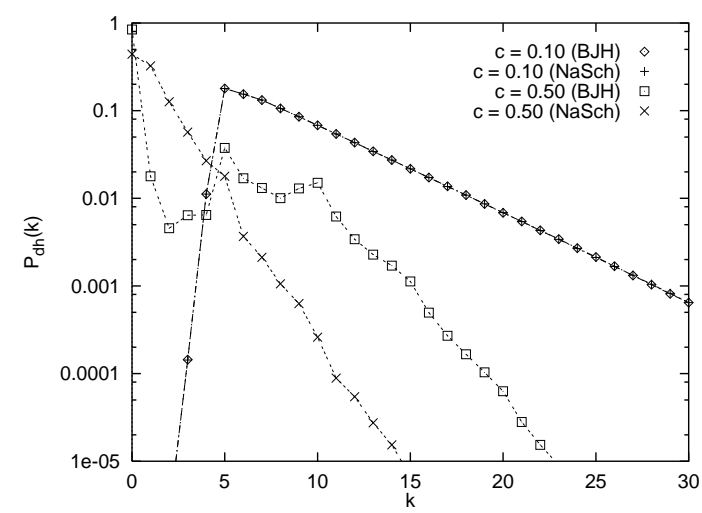

Figure 3: Same as fig.11, except that $V_{\max }=5$ and the lines are merely guides to the eye.

BJH model with $V_{\max }=5$ are shown in fig. 3. The common feature of the DH distributions in figs. 1.2, and 3 is that for $c=0.1$, which corresponds to free-flowing traffic, DH smaller than $V_{\max }$ are strongly suppressed, while beyond $V_{\max }$ the DH distribution decays exponentially. The density $c=0.5$ is sufficiently high so that there is a very high probability for a vehicle to be stuck in a jam; this is consistent with the maximum of $P_{d h}(k)$ located at $k=0$. Similar peaks at $k=0$ have also been observed earlier 17 in the $\mathrm{DH}$ distribution in the NaSch model. What is more interesting is that the $\mathrm{DH}$ distribution in the $\mathrm{BJH}$ model with $V_{\max }=5$ exhibits 
peaks also at $k=5$ and $k=10$, i.e., at $V_{\max }$, and $2 V_{\max }$ (albeit of gradually smaller heights). This phenomenon can be understood by considering $p=0$ and recalling that, for the values of the parameters used in our simulation, phase separation of traffic into a "free-flowing" region and a region of jammed vehicles occurs at $c=0.5$. If $p=0$, then, the distances between the vehicles in the free flowing region of traffic are determined by the waiting times of the leading vehicle in a jam. If the leading vehicles leaves the jam in the very first opportunity then its $\mathrm{DH}$ is likely to be $V_{\max }$ whereas its likely $\mathrm{DH}$ is $2 V_{\max }$ if it starts at the next time step because of the "slow-to-start" rule. This perfect arrangement of the vehicles would correspond to delta function-like peaks at $V_{\max }$ and $2 V_{\max }$, etc. but gets smeared out to sharp peaks, due to the fluctuations, if $p$ is nonzero but not too large.

\section{The distribution of jam-sizes}

Within the framework of the COMF theory, the distribution of the sizes of the jams in the BJH model with $V_{\max }=1$ can be written as 20]

$$
\mathcal{P}_{j s}(k)=\mathcal{N}_{j s}[1-\mathcal{P}(0)][\mathcal{P}(0)]^{k-1}[1-\mathcal{P}(0)]
$$

where $\mathcal{N}_{j s}$ is a normalization coefficient. Since, the condition $\sum_{k} \mathcal{P}_{j s}(k)=1$ of normalization leads to $\mathcal{N}_{j s}=[1-\mathcal{P}(0)]^{-1}$, the normalized distribution of the jam sizes is given by

$$
\mathcal{P}_{j s}(k)=[1-\mathcal{P}(0)][\mathcal{P}(0)]^{k-1}
$$

In the limit $p_{s} \rightarrow 0$,

$$
\mathcal{P}_{j s}(k) \rightarrow \frac{y}{c}\left(1-\frac{y}{c}\right)^{k-1}
$$

which is identical to the jam-size distribution in the NaSch model with $V_{\max }=1$ where $y$ is given by the equation (10).

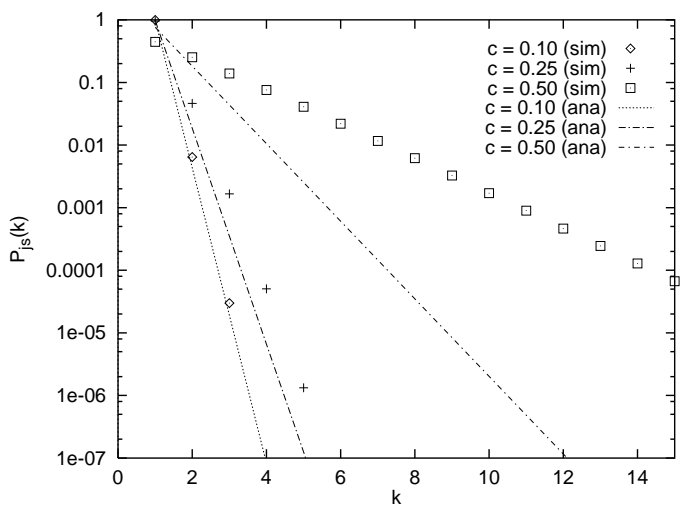

Figure 4: The distribution of jam-sizes in the BJH model with $V_{\max }=1$ are shown on a semi-log plot for the same values of $c, p$ and $p_{s}$ as in fig. 1,2. The lines are obtained from the approximate analytical expression (12) for $\mathcal{P}_{j s}(k)$ while the discrete data points have been obtained from computer simulation.

The jam-size distribution for the BJH model is well approximated by the expression (12) at low densities of the vehicles as is evident from the fig. (1 where comparison has been made with the corresponding numerical data obtained from computer simulation of the BJH model with the same set of parameters. However, the approximate expression (12) deviates more and more from the computer 


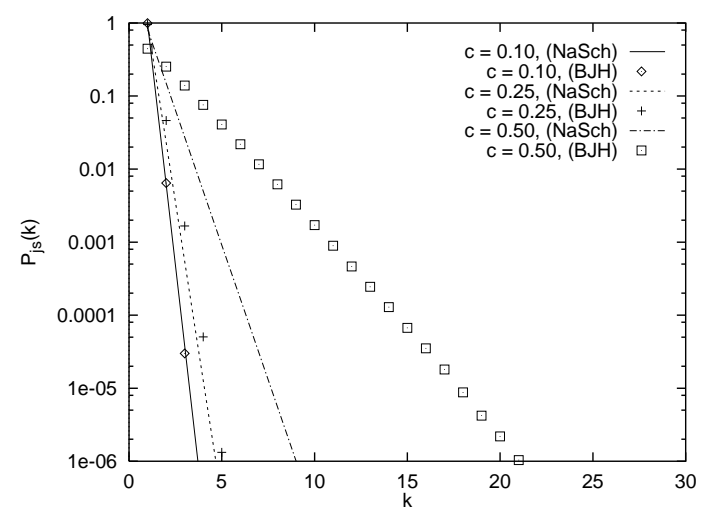

Figure 5: The distributions of jam-sizes in the BJH model and the NaSch model, both with $V_{\max }=1$ and $p=0.05$, are shown on a semi-log plot for three different values of the density of vehicles $c$. The lines are obtained from the exact analytical expressions for $\mathcal{P}_{j s}(k)$ in the NaSch model while the discrete data points have been obtained from computer simulation of the BJH model with $p_{s}=0.50$.

simulation data with increasing density for the densities we took into account. A comparison between the jam-size distributions in the NaSch and BJH models, both for $V_{\max }=1$ and $p=0.05$, is made in fig. 5 . This comparison establishes that the difference in the jam-size distributions in these two models becomes wider with the increase of vehicle density $c$. At a high density of $c=0.5$, for example, long jams are much more probable in the BJH model than in the NaSch model. A few typical

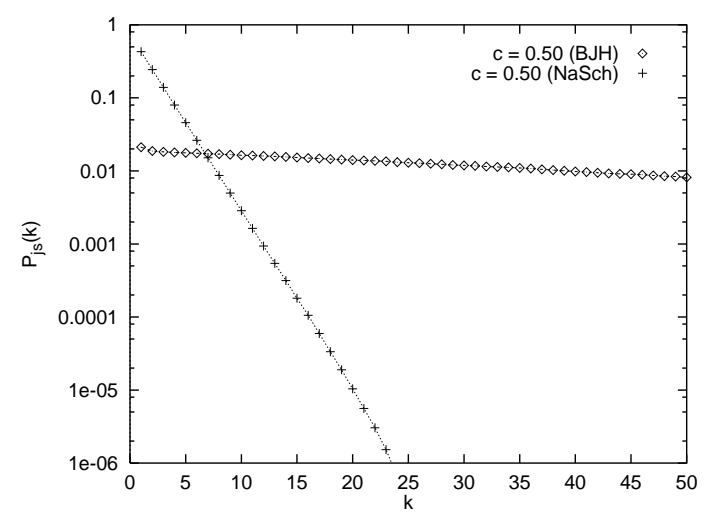

Figure 6: Same as fig. 4 , except that $V_{\max }=5$ and the lines are merely guides to the eye.

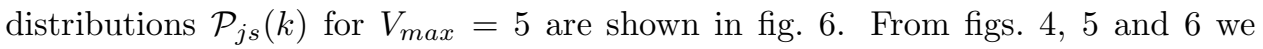
conclude that, for both $V_{\max }=1$ and $V_{\max }=5$, the jam size distributions $\mathcal{P}_{j s}(k)$ decay exponentially with the jam-size $k$.

\section{The distribution of gaps between successive jams}

\subsection{A hybrid approach}

Let us use a three-state variable $s$ to describe the state of a site; $s=-1$ if the site is empty, $s=0$ if the site is occupied by a vehicle with instantaneous speed $V=0$ and $s=1$ if the site is occupied by a vehicle with instantaneous speed $V=1$ [17]. 
Note that this variable $s$ does not distinguish between the slow-to-start vehicles and other vehicles which have instantaneous speed $V=0$. We denote the probability of a configuration immediately after the slow-to-start stage by the symbol $P(a, b \cdots)$ while that immediately after the randomization stage by $p(c, d \cdots)$ and use the sequence $5-1-2-3-4$ of the steps of the update rules, instead of $1-2-3-4-5$.

In terms of the state variable $s$, a 2 -cluster - like approximate decomposition of the probability distribution of the gaps between successive jams leads to the expression

$$
\mathcal{P}_{j g}(k)=\mathcal{N}_{j g}\left[\sum_{\left\{s_{i}= \pm 1\right\}} p_{2}\left(\underline{0} \mid s_{1}\right) p_{2}\left(\underline{s_{1}} \mid s_{2}\right) \cdots p_{2}\left(\underline{s_{k-1}} \mid s_{k}\right) p_{2}\left(\underline{s_{k}} \mid 0\right)\right] \quad \text { for } k \geq 1
$$

where $\mathcal{N}_{j g}$ is a normalization coefficient and the conditional probabilities $p_{2}(\underline{a} \mid b)$ are defined through the equation

$$
p_{2}(\underline{a} \mid b)=\frac{p_{2}(a, b)}{p_{2}(a, 0)+p_{2}(a,-1)+p_{2}(a, 1)} .
$$

Let us now define the $2 \times 2$ transfer matrix $T$ with the following elements:

$$
\begin{array}{llrl}
T[1,1] & =p_{2}(\underline{1} \mid 1), & & T[1,2]=p_{2}(\underline{1} \mid-1), \\
T[2,1]=p_{2}(\underline{-1} \mid 1), & & T[2,2]=p_{2}(\underline{-1} \mid-1) .
\end{array}
$$

In terms of this transfer matrix, the expression (13) for $P_{j g}(k)$ can be recast as

$$
\begin{aligned}
\mathcal{P}_{j g}(k) & =\mathcal{N}_{j g}\left[p_{2}(\underline{0} \mid 1) T^{k-1}[1,1] p_{2}(\underline{1} \mid 0)+p_{2}(\underline{0} \mid-1) T^{k-1}[2,1] p_{2}(\underline{1} \mid 0)\right. \\
& \left.+p_{2}(\underline{0} \mid 1) T^{k-1}[1,2] p_{2}(\underline{-1} \mid 0)+p_{2}(\underline{0} \mid-1) T^{k-1}[2,2] p_{2}(\underline{-1} \mid 0)\right](16)
\end{aligned}
$$

where $T^{k-1}[i, j]$ refers to the $[i, j]$ element of the matrix $T^{k-1}$. It is straightforward to show that

$$
\begin{aligned}
& T^{k-1}[1,1]=\left(\lambda_{1} \lambda_{2}^{k-1}-\lambda_{2} \lambda_{1}^{k-1}\right) /\left(\lambda_{1}-\lambda_{2}\right), \\
& T^{k-1}[1,2]=\left(\lambda_{1}^{k-1}-\lambda_{2}^{k-1}\right) /\left(\lambda_{1}-\lambda_{2}\right), \\
& T^{k-1}[2,1]=\left(\lambda_{1} \lambda_{2}^{k}-\lambda_{2} \lambda_{1}^{k}\right) /\left(\lambda_{1}-\lambda_{2}\right), \\
& T^{k-1}[2,2]=\left(\lambda_{1}^{k}-\lambda_{2}^{k}\right) /\left(\lambda_{1}-\lambda_{2}\right),
\end{aligned}
$$

where $\lambda_{1}$ and $\lambda_{2}$ are the two eigenvalues of the transfer matrix $T$.

As shown in the appendix A, the 2-cluster-like conditional probabilities $p_{2}(\underline{a} \mid b)$ can be related to the quantities like $g, z, \mathcal{P}(0), \mathcal{P}(1), \tilde{\mathcal{P}}(1)$, etc. which occur in the COMF theory. Using these relations we get

$$
\begin{aligned}
T[1,1]= & p_{2}(\underline{1} \mid 1)=0, \\
& T[1,2]=p_{2}(\underline{1} \mid-1)=1, \\
T[2,1]= & p_{2}(\underline{-1} \mid 1)=\frac{c g(1-\mathcal{P}(0))}{c[1-\mathcal{P}(0)]+z(1-c)}, \\
T[2,2]= & p_{2}(\underline{-1} \mid-1)=\frac{z(1-c)}{c[1-\mathcal{P}(0)]+z(1-c)},
\end{aligned}
$$

and, hence [35], with $D=c\left[p+q \mathcal{P}(0)+q p_{s} \tilde{\mathcal{P}}(1)\right]$,

$$
\begin{aligned}
\mathcal{P}_{j g}(k) & =\mathcal{N}_{j g} \cdot \frac{c \mathcal{P}(0) g}{D} \cdot \frac{\lambda_{1}^{k-1}-\lambda_{2}^{k-1}}{\lambda_{1}-\lambda_{2}} \cdot \frac{c(1-g)[1-\mathcal{P}(0)]}{c[1-\mathcal{P}(0)]+z(1-c)} \\
& +\left(1-\frac{c \mathcal{P}(0)}{D}\right) \cdot \frac{\lambda_{1}^{k}-\lambda_{2}^{k}}{\lambda_{1}-\lambda_{2}} \cdot \frac{c(1-g)[1-\mathcal{P}(0)]}{c[1-\mathcal{P}(0)]+z(1-c)}
\end{aligned}
$$


where

$$
\begin{aligned}
\lambda_{1,2} & =\frac{1}{2}\left[\frac{z(1-c)}{z(1-c)+c[1-\mathcal{P}(0)]}\right. \\
& \left. \pm \sqrt{\left(\frac{z(1-c)}{z(1-c)+c[1-\mathcal{P}(0)]}\right)^{2}+\frac{4 c g[1-\mathcal{P}(0)]}{z(1-c)+c[1-\mathcal{P}(0)]}}\right]
\end{aligned}
$$

are the eigenvalues of the $T$ matrix in this approximation. From equations (25) and (26) we find that, in the limit $p_{s} \rightarrow 0$,

$$
\lambda_{1,2} \rightarrow \frac{1}{2}\left[\left(1-\frac{y}{1-c}\right) \pm \sqrt{\left(1-\frac{y}{1-c}\right)^{2}+4\left(\frac{y}{c(1-c)}-1\right)}\right],
$$

and

$$
\mathcal{P}_{j g}(k) \rightarrow \frac{p y^{2} c\left(\lambda_{1}^{k}-\lambda_{2}^{k}\right)+q y^{2}(c-y)\left(\lambda_{1}^{k-1}-\lambda_{2}^{k-1}\right)}{c^{2}(1-c)\left(\lambda_{1}-\lambda_{2}\right)}
$$

which is the jam-gap distribution in the NaSch model for $V_{\max }=1$ [17].

The probabilities like $p_{2}\left(s_{k-1}, s_{k}\right)$ which appeared in the intermediate steps of the calculations in this subsection are not true 2-cluster probabilities in the usual sense of the term [13]. The final expression for the distribution $P_{j g}(k)$ involves only quantities which we compute entirely within the framework of the COMF theory. Therefore, this whole approach may be called a hybrid approach. We shall compare the expression (25) for $\mathcal{P}_{j g}(k)$ as well as the corresponding expression derived in the next subsection following the true 2-cluster approach with the data obtained from computer simulation at the end of this section.

\subsection{The 2-cluster approach}

We now derive the distribution $P_{j g}(k)$ using the true 2-cluster approximation. Let us now allow the state variable $s$ to take one of the four possible values, namely, $s=-1$ corresponding to an empty site, $s=1$ corresponding to a site occupied by a vehicle with instantaneous speed $V=1$ while $s=0$ and 2 correspond to a site occupied by, respectively, a non-slow-to-start vehicle with $V=0$ and a slowto-start vehicle (also with $V=0$ ). In terms of this new definition of the variable $s$, we can write the distribution of the gaps between the jams in the BJH model with $V_{\max }=1$ as

$$
\mathcal{P}_{j g}(k)=\mathcal{N}_{j g}^{\prime} \sum_{\sigma, \sigma^{\prime}=0,2} \sum_{\left\{s_{i}= \pm 1\right\}} C_{2}\left(\underline{\sigma} \mid s_{1}\right) C_{2}\left(\underline{s_{1}} \mid s_{2}\right) \cdots C_{2}\left(\underline{s_{k-1}} \mid s_{k}\right) C_{2}\left(\underline{s_{k}} \mid \sigma^{\prime}\right)
$$

where $\mathcal{N}_{j g}^{\prime}$ is a normalization coefficient and $C_{2}(\underline{\mathrm{a}} \mid b)$ are the true conditional 2cluster probabilities. In terms of the transfer matrix $T$, whose elements are given by the equation

$$
\begin{aligned}
T[1,1] & =C_{2}(\underline{1} \mid 1), & T[1,-1] & =C_{2}(\underline{1} \mid-1), \\
T[-1,1] & =C_{2}(\underline{-1} \mid 1), & T[-1,-1] & =C_{2}(\underline{-1} \mid-1),
\end{aligned}
$$

the equation (27) can be written as

$$
\mathcal{P}_{j g}(k)=\mathcal{N}_{j g}^{\prime} \sum_{\left\{\sigma, \sigma^{\prime}=0,2\right\}} \sum_{s, s^{\prime}= \pm 1} C_{2}(\underline{\sigma} \mid s) T^{k-1}\left[s, s^{\prime}\right] C_{2}\left(\underline{s}^{\prime} \mid \sigma^{\prime}\right) .
$$

For convenience we have labeled the elements of $T$ by $(1,1),(1,-1),(-1,1),(-1,-1)$ instead of $(1,1),(1,2),(2,1),(2,2)$. As before, $T^{k-1}[i, j]$ refers to the $[i, j]$ element of 
the matrix $T^{k-1}$ and are given by the equations (17-20). We compute the 2-cluster probabilities as outlined in appendix B and substituting in equation (29) get the distribution of gaps between successive jams in the BJH model with $V_{\max }=1$.

Just as in the case of the jam-size distribution, the agreement of expressions

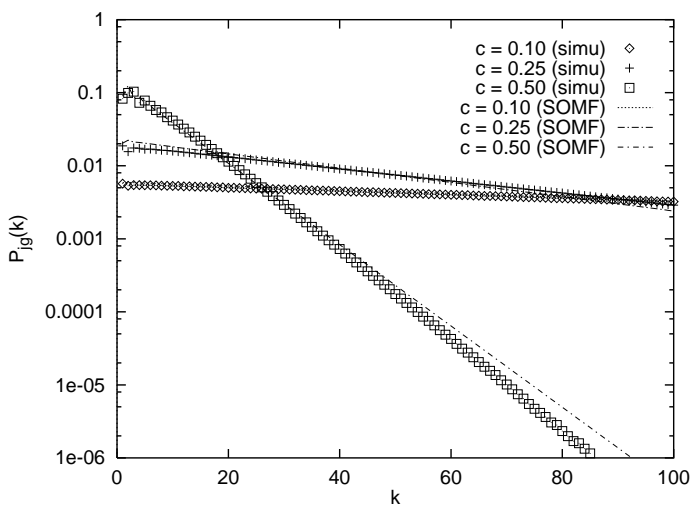

Figure 7: The distribution of sizes of gaps between successive jams in the BJH model with $V_{\max }=1$ are shown on a semi-log plot for the same values of $c, p$ and $p_{s}$ as in fig. 1.2. The lines are obtained from the approximate analytical expression (29) for $\mathcal{P}_{j g}(k)$ while the discrete data points have been obtained from computer simulation.

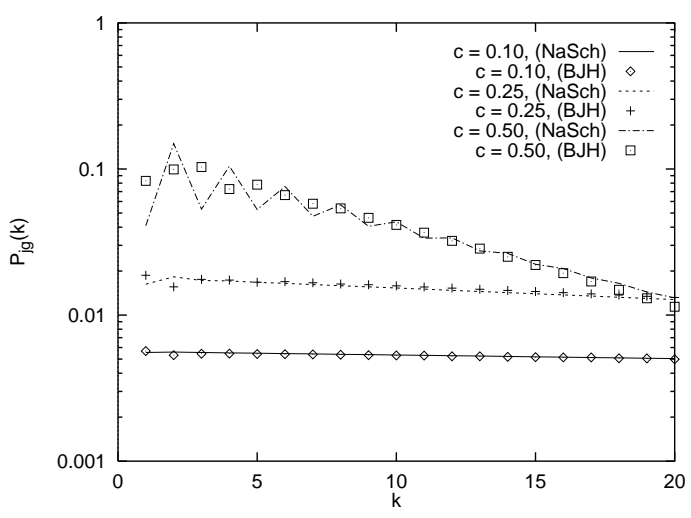

Figure 8: The distributions of the gaps between successive jams in the BJH model and the NaSch model, both with $V_{\max }=1$ and $p=0.05$, are shown on a semi-log plot for three different values of the density of vehicles $c$. The lines are obtained from the exact analytical expressions for $\mathcal{P}_{j g}(k)$ in the NaSch model while the discrete data points have been obtained from computer simulation of the BJH model with $p_{s}=0.50$.

(25) and (29) with the corresponding computer simulation data is better when the vehicle density is lower (fig. 1). We have found that, at lower densities, the prediction of the hybrid approach (i.e., the equation (25)), rather than that of the true 2-cluster approximation (i.e., the equation (29), is in better agreement with the corresponding computer simulation data. However, beyond some intermediate density, the results of the true 2-cluster approximation are found to be closer to the computer simulation data than the corresponding results obtained in the hybrid approach. It is interesting to note that, for the values of the set of parameters chosen for fig. 7, there is very little difference between the jam-gap distributions in the NaSch and BJH models (see fig. 8). 


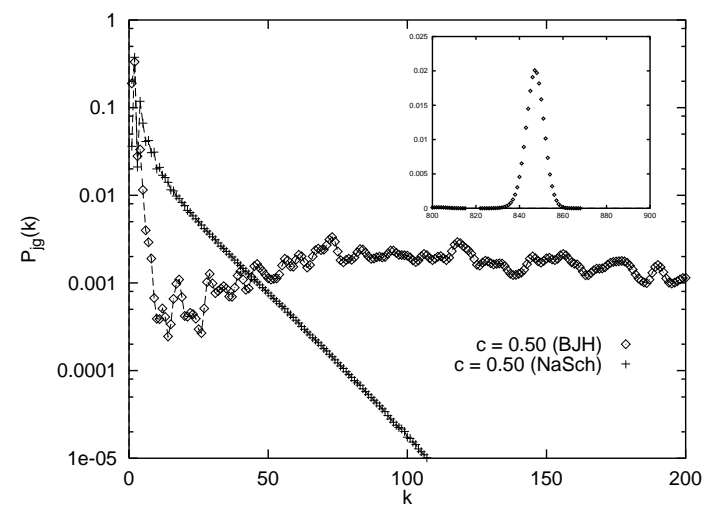

Figure 9: Same as fig. 7, except that $V_{\max }=5$ and the lines are merely guides to the eye. The inset shows the local maximum at $\bar{L}_{f}(c)$.

Comparing the curves in fig. 7,8 8 with the corresponding distributions for $V_{\max }=$ 5 , shown in fig. 9, we conclude that the distribution $\mathcal{P}_{j g}(k)$ decays exponentially with the gap $k$ between the successive jams for all finite $V_{\max }$, as far as small distances are considered. Nevertheless $\mathcal{P}_{j g}(k)$ allows to identify macroscopic free flow regimes. In particular, we found for $v_{\max }=5, p_{s}=0.5$, and $p=0.05$ a local maximum of the distance distribution at $\bar{L}_{f}(c)=L-\bar{L}_{j}(c)$, where $\bar{L}_{f}(c)$ denotes the average size of the free flow regime at a given density, and $\bar{L}_{f}(c)$ the typical size of the macroscopic jam. Therefore the jam-gap distribution is well suited to identify at least the macroscopic free flow regime of a phase separated high density state.

\section{The TH distribution}

We label the position of the detector by $j=0$, the site immediately in front of it by $j=1$, and so on. For the convenience of analytical calculations of the $\mathrm{TH}$ distribution in the steady state of the BJH model with $V_{\max }=1$, we assume the sequence of steps $2-3-4-5-1$, just as in the corresponding calculation for the NaSch model [18, 19] the sequence of steps was assumed to be $2-3-4-1$.

Suppose, $\mathcal{P}_{m}\left(t_{1}\right)$ is the probability that the FV takes time $t_{1}$ to reach the detector moving from its initial position, where it was located when the clock at the detector site was set to $t=0$, after the LV just left the detector site. Suppose, after reaching the detector site, the FV waits there for $\tau-t_{1}$ time steps, either because of the presence of another vehicle in front of it or because of its own random braking; the probability for this event is denoted by $Q\left(\tau-t_{1} \mid t_{1}\right)$. Then, the distribution $\mathcal{P}_{t h}(\tau)$, of the $\mathrm{TH} \tau$, is given by

$$
\mathcal{P}_{t h}(\tau)=\sum_{t_{1}=1}^{\tau-1} \mathcal{P}_{m}\left(t_{1}\right) Q\left(\tau-t_{1} \mid t_{1}\right)
$$

or, equivalently, by

$$
\mathcal{P}_{t h}(\tau)=\sum_{t_{w}=1}^{\tau-1} \mathcal{P}_{m}\left(\tau-t_{w}\right) Q\left(t_{w} \mid \tau-t_{w}\right)
$$

where $t_{w}$ is the waiting time at the detector site.

We calculate $\mathcal{P}_{t h}(\tau)$ for $V_{\max }=1$ analytically using the equation (30). In order to calculate $\mathcal{P}_{m}\left(t_{1}\right)$ we consider those spatial configurations at $t=0$ from which the 
FV can reach the detector site within $t_{1}$ time steps. This implies that one needs to consider configurations up to a maximum separation of $t_{1}$ sites from the detector site so that the FV vehicle can reach the detector site even from the farthest point with $t_{1}$ hops. Thus, the configurations of interest are of the form

$$
(\underbrace{1,-1, \ldots .,-1}_{n \text { times }} \mid \underline{-1})
$$

where $n=1,2, \ldots, t_{1}$. The underlined -1 implies that we have to find the conditional steady-state probability for the given configuration subject to the condition that the underlined site (detector site) has just become empty. In the 2-cluster approximation, this probability, $\Pi(n)$, is given by

$$
\Pi(n)=P_{2}(1 \mid \underline{-1})\left\{P_{2}(-1 \mid \underline{-1})\right\}^{n-1} .
$$

where, the 2-cluster steady-state probabilities are given by

$$
P_{2}(1 \mid \underline{-1})=\frac{c}{d}\left[1-\mathcal{P}_{0}-p_{s} \tilde{\mathcal{P}}(1)\right]
$$

and

$$
P_{2}(-1 \mid \underline{-1})=z=\frac{p g}{q \bar{g}}
$$

For all configurations with $t_{1}>n$, the vehicle has to stop $\left(t_{1}-n\right)$ times in order to reach the detector site after exactly $t_{1}$ time steps. Equivalently, $t_{1}-1$ time steps must elapse in crossing $n_{1}$ bonds (as the vehicle must certainly hop across the bond connecting the detector site with its preceding site at the last time step); the number of ways in which $t_{1}-1$ time steps can be distributed among $n-1$ bonds is $\left(\begin{array}{c}t_{1}-1 \\ n-1\end{array}\right)$. Thus,

$$
\mathcal{P}_{m}\left(t_{1}\right)=\sum_{n=1}^{t_{1}}\left(\begin{array}{c}
t_{1}-1 \\
n-1
\end{array}\right) \Pi(n) q^{n} p^{t_{1}-n}
$$

as a factor $q$ for every forward step and a factor of $p$ for every halt must be included. Substituting (32) and (33) into equation (31) and using the corresponding expression for $\Pi(n)$ in (34) we get

$$
\mathcal{P}_{m}\left(t_{1}\right)=\mathcal{N}_{t}\left(1-\mathcal{P}_{0}-p_{s} \tilde{\mathcal{P}}(1)\right) \frac{q c}{d}\left(\frac{p}{\bar{g}}\right)^{t_{1}-1}
$$

where $\mathcal{N}_{t}$ is the normalization factor. The normalization condition $\sum_{t_{1}=1}^{\infty} \mathcal{P}_{m}\left(t_{1}\right)=$ 1 leads to

$$
\mathcal{P}_{m}\left(t_{1}\right)=\frac{\bar{g}-p}{\bar{g}}\left(\frac{p}{\bar{g}}\right)^{t_{1}-1}
$$

or, equivalently,

$$
\mathcal{P}_{m}\left(\tau-t_{w}\right)=\frac{\bar{g}-p}{\bar{g}}\left[1-\frac{\bar{g}-p}{\bar{g}}\right]^{\tau-t_{w}-1}
$$

It is straightforward to verify that in the limit $p_{s} \rightarrow 0,(\bar{g}-p) / \bar{g} \rightarrow(q y / d)$ and, hence, from the expression (36) we find that

$$
\mathcal{P}_{m}\left(t_{1}\right) \rightarrow \frac{q y}{d}\left[1-\frac{q y}{d}\right]^{t_{1}-1}
$$

which is the corresponding expression for the NaSch model [18]. 
Next, let us derive an analytical expression for $Q\left(t_{w} \mid \tau-t_{w}\right)$. When the FV arrives at the detector site exactly $\tau-t_{w}$ time steps after the departure of the LV, the $\mathrm{LV}$ can be at any of the sites labeled by $1,2, \ldots \tau-t_{w}+1$. We consider two situations separately, namely, (i) the LV is not at site '1' when the FV arrives at the detector site, and (ii) the LV is still at site ' 1 ' when the FV arrives at the detector site. Let us first consider the situation (i). The probability that the LV stays at the site '1', i.e., it does not move in any of the $\tau-t_{w}$ time steps $=(\bar{g})^{\tau-t_{w}}$. Therefore, the probability that the $\mathrm{LV}$ is not at site ' 1 ' $=1-(\bar{g})^{\tau-t_{w}}$. If the LV is not at site ' 1 ' then the probability that the FV halts at the detector site for exactly $t_{w}$ time steps is $p^{t_{w}-1} q$ because it should halt due to random braking for exactly $t_{w}-1$ time steps and move at the last time step. Thus, the contribution to $Q\left(t_{w} \mid \tau-t_{w}\right)$ coming from all those situations where the LV is not at site ' 1 ' when the FV reaches the detector site is

$$
\left[1-(\bar{g})^{\tau-t_{w}}\right] p^{t_{w}-1} q
$$

Let us now calculate the contribution to $Q\left(t_{w} \mid \tau-t_{w}\right)$ from those situations which are of type (ii), i.e., where the LV is at site ' 1 ' when the FV reaches the detector site. So, the LV will have to move so that the FV is able to leave the detector site (and move forward) after $t_{w}$ time steps. Suppose, the LV moves from '1' after $k$ time steps $\left(1 \leq k \leq t_{w}-1\right)$, then the FV will have to stay at the detector site for the next $t_{w}-1-k$ time steps either because of random braking or because of the slow-to-start rule and, then, move forward at the last time step. Note that if $t_{w}=1$, i.e., if the FV stays at the detector site only for one time step, then the site '1' must be empty when the FV reaches the detector site; the corresponding contribution to $Q\left(t_{w} \mid \tau-t_{w}\right)$ has already been included in (I). The calculation of $Q\left(t_{w} \mid \tau-t_{w}\right)$ for all $\tau \geq 2$ is quite non-trivial. Suppose, the LV moves from the site ' 1 ' after $k$ time steps and $k<t_{w}-1$. Then the FV can stay at the detector site during the immediately next time step with probability $p_{s}+q_{s} p$ and for the remaining $t_{w}-2-k$ time steps with probability $p$ each; in the last step it must move forward with probability $q$. There is, however, yet another possibility. When the LV moves from ' 1 ' after $t_{w}-1$ time steps, then the FV has to move forward in the last time step; the corresponding probability being $q q_{s}$. Thus, the contribution to all these situations of type (ii) is

$$
\begin{gathered}
(\bar{g})^{\tau-t_{w}} q g\left[\sum_{k=1}^{t_{w}-2}(\bar{g})^{k-1} p^{t_{w}-2-k}\left(p_{s}+q_{s} p\right)+(\bar{g})^{t_{w}-2} q_{s}\right] \\
=(\bar{g})^{\tau-t_{w}} q g\left[\frac{\alpha(\bar{g})^{t_{w}-2}-\beta p^{t_{w}-2}}{\bar{g}-p}\right]
\end{gathered}
$$

where $\alpha=p_{s}+q_{s} \bar{g}$ and $\beta=p_{s}+q_{s} p$. Thus, combining (I) and (II) we find

$$
Q\left(t_{w} \mid \tau-t_{w}\right)=\left[1-(\bar{g})^{\tau-1}\right] q \quad \text { for } \quad t_{w}=1
$$

and

$$
\begin{aligned}
Q\left(t_{w} \mid \tau-t_{w}\right) & =\left[1-(\bar{g})^{\tau-t_{w}}\right] p^{t_{w}-1} q \\
& +(\bar{g})^{\tau-t_{w}} q g\left[\frac{\alpha(\bar{g})^{t_{w}-2}-\beta p^{t_{w}-2}}{\bar{g}-p}\right] \quad \text { for } t_{w} \geq 2
\end{aligned}
$$

In the limit $p_{s} \rightarrow 0$,

$$
Q\left(\tau-t_{1} \mid t_{1}\right) \rightarrow\left[1-(\bar{g})^{t_{1}}\right] p^{\tau-t_{1}-1} q+(\bar{g})^{t_{1}} g q\left[\frac{(\bar{g})^{\tau-t_{1}-1}-(p)^{\tau-t_{1}-1}}{\bar{g}-p}\right]
$$

which is, indeed, the corresponding expression for $Q\left(\tau-t_{1} \mid t_{1}\right)$ in the NaSch model [19]. 
Finally, using (37) and (38) in (30) and carrying out the summation we get

$$
\begin{aligned}
\mathcal{P}_{t h}(\tau) & =\mathcal{N}_{t h}\left[\frac{\bar{g}-p}{\bar{g}} \cdot \frac{(1 / \bar{g})^{\tau-1}-1}{(1 / \bar{g})-1} \cdot q p^{\tau-2}+\frac{\alpha q g}{\bar{g}} \cdot \frac{(\bar{g})^{\tau-1}-p^{\tau-1}}{\bar{g}-p}\right. \\
& \left.-(\bar{g}-p) q p^{\tau-2}(\tau-1)-g q \beta p^{\tau-3}(\tau-1)-g q p^{\tau-2}\left(\frac{\alpha}{\bar{g}}-\frac{\beta}{p}\right)\right]
\end{aligned}
$$

where $\mathcal{N}_{t h}$ is the normalization coefficient (which is required as the expression (39) is not exact). From the expression (39) we also find that in the limit $p_{s} \rightarrow 0$,

$$
\begin{gathered}
\mathcal{P}_{t h}(\tau)=\frac{q y}{c-y}\left[1-\frac{q y}{c}\right]^{\tau-1}+\frac{q y}{d-y}\left[1-\frac{q y}{d}\right]^{\tau-1} \\
-\left[\frac{q y}{c-y}+\frac{q y}{d-y}\right] p^{\tau-1}-q^{2}(\tau-1) p^{\tau-2}
\end{gathered}
$$

which is the known exact expression for $\mathcal{P}_{t h}(\tau)$ in the NaSch model 19].

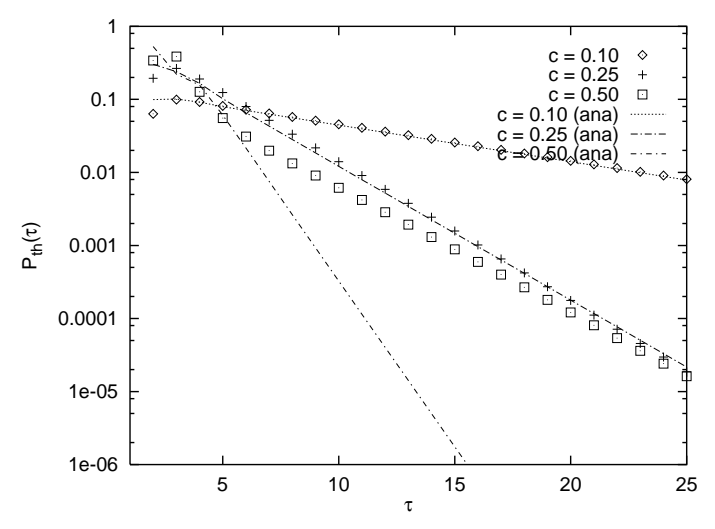

Figure 10: The TH-distribution in the BJH model with $V_{\max }=1$ are shown on a semi-log plot for the same values of $c, p$ and $p_{s}$ as in figs. 1, 2. The lines are obtained from the approximate analytical expression $(82)$ for $\mathcal{P}_{t h}(\tau)$ while the discrete data points have been obtained from computer simulation.

The TH-distribution for the BJH model with $V_{\max }=1$ is well approximated by the expression (39) at low densities of the vehicles but the approximate expression (82) deviates more and more from the computer simulation data with increasing density (fig. 10). A comparison of the $\mathrm{TH}$ distributions in the NaSch and BJH models, both for $V_{\max }=1$ and $p=0.05$, in fig. 11, clearly demonstrates that the larger is the density $c$ the wider is the difference between the TH distributions in these two models. In fact, at a high density, e.g., $c=0.5$, the larger $\mathrm{TH}$ are much more probable in the BJH model than in the NaSch model. This is basically for two reasons. Firstly the slow-to-start rule leads to larger jams compared to the NaSch model at the same density (see fig. 5) and secondly the downstream velocity of the jams is reduced, which also leads to an increase of the typical waiting time for a car in a jam. The computer simulation data for the same densities as in fig. 10,11, but for $V_{\max }=5$ are shown in fig. 12. From the definition of $\mathrm{TH}$ it is clear that, for the NaSch model as well as the BJH model, the TH distributions must vanish for $\tau<2$ if $V_{\max }=1$ but need not do so if $V_{\max }>1$ [18]. Except for this difference, at low densities, e.g., $c=0.1$ the $\mathrm{TH}$ distributions $\mathcal{P}_{t h}(\tau)$ for $V_{\max }=1$ is qualitatively similar to that for $V_{\max }=5$; both exhibit a maximum and fall exponentially with $\tau$ beyond the most-probable TH. But, there are some qualitative differences in the $\tau$-dependence of $\mathcal{P}_{t h}(\tau)$ for $V_{\max }=1$ and that for 


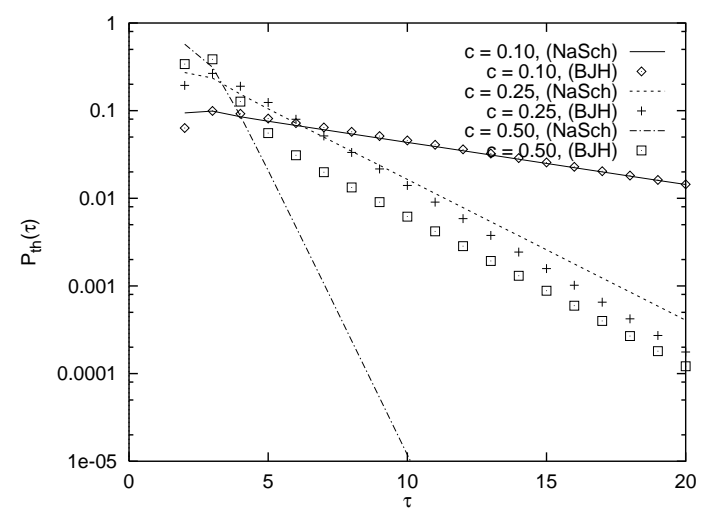

Figure 11: The TH distributions in the BJH model and the NaSch model, both with $V_{\max }=1$ and $p=0.05$, are shown on a semi-log plot for three different values of the density of vehicles $c$. The lines are obtained from the exact analytical expressions for $\mathcal{P}_{t h}(k)$ in the NaSch model while the discrete data points have been obtained from computer simulation of the BJH model with $p_{s}=0.50$.

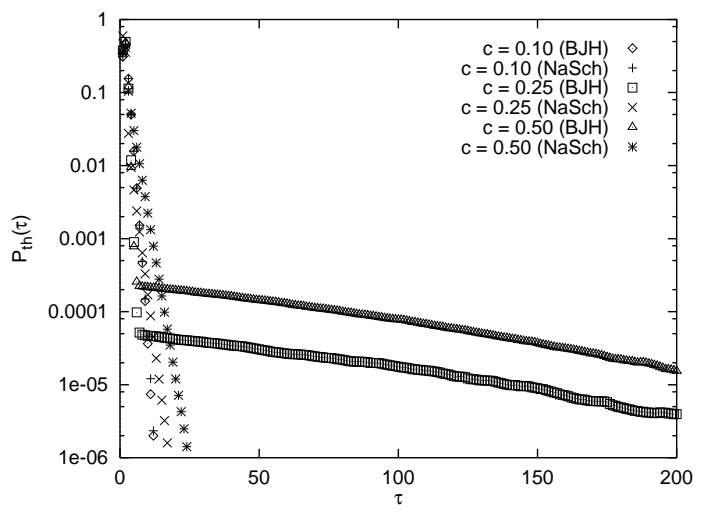

Figure 12: Same as fig. 10, except that $V_{\max }=5$.

$V_{\max }=5$ in the BJH model. Beyond the most probable $\mathrm{TH}$, the $\mathrm{TH}$ distribution for $V_{\max }=1$ can be fitted to a exponentially decaying function with a single decay rate. On the other hand, for $V_{\max }=5$, there are two regimes of $\tau$ : in the small $\tau$ regime, which corresponds to the "free-flowing" regions of traffic, $\mathcal{P}_{t h}(\tau)$ can be fitted to an exponentially decaying function (with a fast rate of decay) whereas in the large $\tau$ regime, which corresponds to the jammed region of traffic, $\mathcal{P}_{t h}(\tau)$ can be fitted also to an exponentially-decaying function (with a very slow rate of decay). This phenomenon of two different regimes of $\tau$, which arises from the phase separation in the BJH model, is absent in the NaSch model [18].

\section{Regime of validity of the analytical expressions}

In the preceding section we have observed that the analytical expressions derived for the BJH model with $V_{\max }=1$ are is good agreement with the numerical data from our computer simulation if the density of vehicles is not high. However, the higher is the vehicle density the stronger are those correlations which have been neglected in our calculations within the COMF approximation and the 2-cluster approximation; these correlations lead to increasing deviation of the analytical result, from the corresponding simulation data, with increasing density of the vehicles. We now 
make a quantitative estimation of the regime of validity of our analytical results derived in this paper.

For the NaSch model, the 2-cluster approximation, which gives exact results for $V_{\max }=1$, is known to be equivalent to the car-oriented mean-field (COMF) theory, which neglects the correlations of the type

$$
G_{n, m}=\mathcal{P}(n, m)-\mathcal{P}(n) \mathcal{P}(m)
$$

where $\mathcal{P}(n, m)$ is the joint probability of finding a $\mathrm{DH} m$ followed immediately by a $\mathrm{DH} n$. On the other hand, the COMF does not give exact results for the BJH model [28] because of non-vanishing correlations of this type. Therefore, we

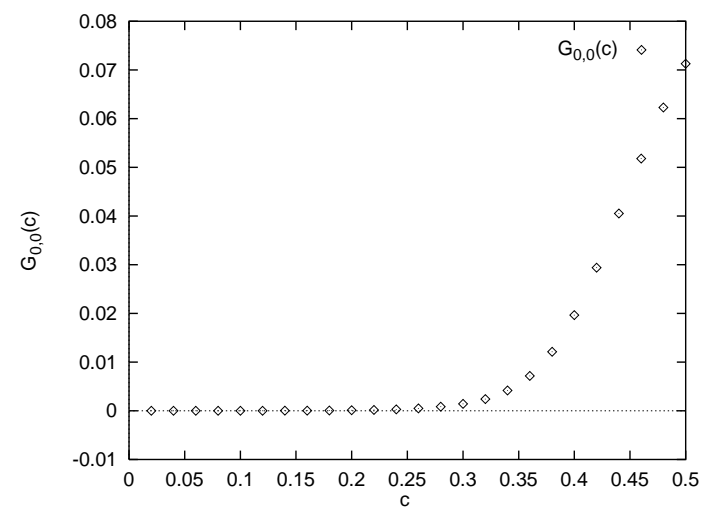

Figure 13: The correlation function $G_{0,0}(c)$ in the BJH model with $V_{\max }=1$ is plotted against $c$ for the same values of the parameters $p$ and $p_{s}$ as in the figs. 1 , 2, 果, 年, 8, 10, and 11 .

have explicitly computed the correlation $G_{0,0}$ as a function of the vehicle density, $c$, for the BJH model with $V_{\max }=1$ through computer simulation (fig. 13). This correlation falls exponentially with decreasing density and can be neglected if $c$ is not larger than about 0.3. This is consistent with all the results summarized in figs. 1, 2, 1, 5, 7, 8, 10, and 11, where we have observed reasonably good agreement between the analytical results and computer simulations for $c=0.10$ and 0.25 but not for $c=0.50$.

\section{Summary and conclusion}

There are, at present, several different CA models, each providing description of a range of traffic phenomena for which it has been specifically developed. Here we have considered the BJH model [25] which incorporates in a collective way, through the stochastic parameter $p_{s}$, a specific realistic aspect of individual driving habits into the minimal CA model suggested in the pioneering work of Nagel and Schreckenberg [11].

In this paper we have investigated in detail the spatio-temporal organization of the traffic in the BJH model [25] by calculating the distributions of $\mathrm{DH}$, $\mathrm{TH}$, jam sizes and gaps between jams. For $V_{\max }=1$, we have computed the $\mathrm{DH}$ distribution using the COMF approach. We have derived analytical expressions for the distributions of jam-sizes and gaps between jams as well as that of the $\mathrm{TH}$ distribution in the BJH model with $V_{\max }=1$ by a combination of COMF theory and a 2-cluster approximation within the SOMF theory. We have compared these approximate results with high quality numerical data obtained from our computer 
simulations. For $V_{\max }>1$, we have computed all the above mentioned distributions only via extensive computer simulations.

We have analyzed the data for two different regimes, namely, $p_{s} \ll p$ and $p_{s} \gg$ $p$. In the regime $p_{s} \ll p$, there is hardly any significant difference between the analytical results and computer simulation data for the BJH model. In the regime $p_{s} \gg p$, we have found, in general, good agreement between analytical results and computer simulation data for $V_{\max }=1$ when the density of vehicles is not too high. However, the higher is the density the wider is the difference between our analytical results and computer simulation data. This is a consequence of the fact that the COMF approximation as well as the 2-cluster SOMF approximation made for our analytical calculations neglect some correlation which become non-negligible beyond a certain range of vehicle density. In order to directly establish this fact we have made a quantitative estimation of the regime of validity of our analytical results by computing an appropriate correlation function.

In order to elucidate the effects of the slow-to-start rule on the spatio-temporal organization of the traffic, for each set of values of the parameters $V_{\max }, p, c$, we have compared all our numerical data for the BJH model $\left(p_{s} \neq 0\right)$ also with the corresponding data for the NaSch model $\left(p_{s}=0\right)$. From this comparison, some novel features of the spatio-temporal organization of vehicles in the BJH model have emerged.

In the BJH model for $V_{\max }=1$ no phase separation takes place and spontaneous jamming is possible also in the free-flow regime. In this sense the model is similar to the NaSch model. This is also reflected in the quantities we have calculated. For $V_{\max }=5$, at sufficiently low densities, i.e., in the free-flow regime, the behavior of the BJH model is also very similar to that of the NaSch model in the corresponding regime. But, at higher densities, phase-separated states appear in the BJH model with $V_{\max }=5$. Therefore, in this case, the jam-gap distribution is qualitatively different from that of the NaSch model, e.g., one finds a local maximum at a macroscopic distance corresponding to the length of the free-flow regime. On the other hand, the jam-size distribution is not a good indicator of the appearance of this phase separation because the large "jam", which is formed in this phase-separated regime contains 'holes' whereas, according to our definition, only a compact cluster of vehicles with $V=0$ qualifies as a single jam.

Measurements on real traffic have shown the existence of metastable states [36, 37. These are related to the phase separation 27] found here and are an indication for the importance of slow-to-start effects in real traffic. Slow-to-start rules lead to a reduction of the outflow $J_{\text {out }}$ from a jam compared to the maximal possible flow $J_{\max }$ [27]. Experimentally one finds $J_{\max } / J_{\text {out }} \approx 1.5$ [36, 37]. In the BJH model for fixed $V_{\max }$ this ratio is determined by $p$ and $p_{s} . p_{s}$ also determines the velocity of jam fronts. Therefore it is possible to tune these parameters in order to get a realistic description of jam properties.

Acknowledgments: It is our pleasure to thank D. Stauffer for a critical reading of the manuscript and for useful suggestions. One of the authors (DC) would like to thank International Center for Theoretical Physics, Trieste, for hospitality, where a part of the work was done. This work is supported, in part, by the SFB341 Köln-Aachen-Jülich. 


\section{A Details of the hybrid approach}

We now calculate the 2-cluster-like probabilities $p_{2}(a, b)$ involved in the hybrid approach to the BJH model with $V_{\max }=1$ immediately after the randomization stage from which one can obtain the conditional probabilities $p_{2}(\underline{a} \mid b)$ etc.

-calculation of $p_{2}(-1,-1)$ :

If the configuration of a pair of nearest-neighbor sites is $(-1,-1)$ after the acceleration stage, it does not change during the slow-to-start stage, blockage stage and the randomization stage and this observation leads to the relation $p_{2}(-1,-1)=$ $P_{2}(-1,-1)$. Using the 2-cluster-like decomposition, we write

$$
\mathcal{P}(n)=P_{2}(\underline{+1} \mid-1)\left[P_{2}(\underline{-1} \mid-1)\right]^{n-1} P_{2}(\underline{-1} \mid 1)
$$

for arbitrary $n \geq 2$ and, correspondingly,

$$
\mathcal{P}(n+1)=P_{2}(\underline{+1} \mid-1)\left[P_{2}(\underline{-1} \mid-1)\right]^{n} P_{2}(\underline{-1} \mid 1)
$$

which lead to $\mathcal{P}(n+1) / \mathcal{P}(n)=P_{2}(-1 \mid-1)=P_{2}(-1,-1) /\left[P_{2}(-1,1)+P_{2}(-1,0)+\right.$ $\left.P_{2}(-1,-1)\right]$. Using the result $P_{2}(-1,1)+P_{2}(-1,0)+P_{2}(-1,-1)=1-c$ (because the probability that the left site is unoccupied, irrespective of the state of the right site, is $1-c)$ we get $\mathcal{P}(n+1) / \mathcal{P}(n)=P_{2}(-1,-1) /(1-c)$. On the other hand, from the equation (3) we get $\mathcal{P}(n+1) / \mathcal{P}(n)=z$. Therefore,

$$
p_{2}(-1,-1)=P_{2}(-1,-1)=z(1-c)
$$

-calculation of $p_{2}(-1,+1)$ :

If there there is a vehicle at the leading site (on the right) with speed 1 at the end of the randomization stage then it must move forward during the next vehicle movement stage. Moreover, the vacancy on the left (following) site of the pair can be a part of s string of $m$ successive empty sites where $m$ can be any nonzero positive integer. Furthermore, since the DH distribution immediately after the randomization stage is identical to that immediately after the acceleration stage (as no vehicle movement takes place in between these two stages of the same time step of updating)

$$
p_{2}(-1,1)=c g \sum_{m=1}^{\infty}[\mathcal{P}(m)+\tilde{\mathcal{P}}(1)]=c g[1-\mathcal{P}(0)]
$$

where we have utilized the normalization condition (1).

-calculation of $p_{2}(-1,0)$ :

The calculation of $p_{2}(-1,0)$ proceeds exactly in the same manner as in case of $p_{2}(-1,+1)$ except for the fact that the leading site (on the right) is now occupied by a vehicle with speed 0 which, consequently, will be unable to move during the next vehicle movement stage; hence, the expression for $p_{2}(-1,0)$ is obtained from that for $p_{2}(-1,+1)$ replacing $g$ in $p_{2}(-1,+1)$ by $1-g$, i.e.,

$$
p_{2}(-1,0)=c(1-g)[1-\mathcal{P}(0)] .
$$

-calculation of $p_{2}(0,-1)$ :

The configuration $0,-1$ can arise immediately at the end of the randomization stage in three different ways:

(i) the vehicle in the following site (on the left) is one which is a slow-to-start vehicle which, indeed, slows down in the slow-to-start stage; the corresponding contribution to the probability $p_{2}(0,-1)$ is $c p_{s} \tilde{\mathcal{P}}(1)$. (ii) the vehicle in the following site (on the left) is one which is a slow-to-start vehicle which, however does not slow down during the slow-to-start stage but slows down at the later stage of randomization; 
the corresponding contribution to $p_{2}(0,-1)$ is $c q_{s} p \tilde{\mathcal{P}}(1)$. In both the cases (i) and (ii) the site immediately in front of the leading site (on the right) must be occupied in order that the vehicle at the following site of the pair can qualify as a candidate for slow-to-start option. (iii) the vehicle in the following site is not a slow-to-start vehicle and it slows down at the randomization stage. Moreover, in this case, the empty leading site can be a part of string of $m$ successive empty sites where $m$ can be any non-zero positive integer; the corresponding contribution to $p_{2}(0,-1)$ is $c p\left[\sum_{m=1}^{\infty} \mathcal{P}(m)\right]=c p[1-\mathcal{P}(0)-\tilde{\mathcal{P}}(1)]$. Therefore, adding all the three contributions we get

$$
p_{2}(0,-1)=c\left[p_{s} \tilde{\mathcal{P}}(1)+q_{s} p \tilde{\mathcal{P}}(1)+p(1-\mathcal{P}(0)-\tilde{\mathcal{P}}(1))\right] .
$$

-calculation of $p_{2}(0,0)$ :

The configuration 0,0 can be obtained immediately after the randomization stage if and only if it was $+1,+1$ immediately after the acceleration stage. The facts (i) that the following site is occupied by a vehicle, (ii) that there is no empty site in front of the following vehicle, and (iii) that the leading vehicle cannot move during the vehicle movement stage (because of its speed being 0 at that stage) lead to

$$
p_{2}(0,0)=c(1-g) \mathcal{P}(0)
$$

-calculation of $p_{2}(0,+1)$ :

We now apply the same arguments as those in the calculation of $p_{2}(0,0)$ except that now the leading vehicle will move during the next vehicle movement stage; therefore, replacing $1-g$ in the expression for $p_{2}(0,+1)$ by $g$ we get

$$
p_{2}(0,+1)=\operatorname{cg} \mathcal{P}(0) \text {. }
$$

-calculation of $p_{2}(1,-1)$ :

The configuration $+1,-1$ can arise immediately at the end of the randomization stage in two different ways:

(i) the vehicle in the following site (on the left) is one which is a slow-to-start vehicle which, however neither slows down during the slow-to-start stage nor slows down at the later stage of randomization; the corresponding contribution to $p_{2}(+1,-1)$ is $c q_{s} q \tilde{\mathcal{P}}(1)$. In this case (i) the site immediately in front of the leading site (on the right) must be occupied in order that the vehicle at the following site of the pair can qualify as a candidate for slow-to-start option. (ii) the vehicle in the following site is neither a slow-to-start vehicle and nor does it slow down at the randomization stage. Moreover, in this case, the empty leading site can be a part of string of $m$ successive empty sites where $m$ can be any non-zero positive integer; the corresponding contribution to $p_{2}(+1,-1)$ is $c q \sum_{m=1}^{\infty} \mathcal{P}(m)=c q[1-\mathcal{P}(0)-\tilde{\mathcal{P}}(1)]$. Therefore, adding the two contributions we get

$$
p_{2}(+1,-1)=c q\left[q_{s} \tilde{\mathcal{P}}(1)+(1-\mathcal{P}(0)-\tilde{\mathcal{P}}(1))\right]
$$

-calculation of $p_{2}(+1,0)$ and $p_{2}(+1,+1)$ : Since the speed of a following vehicle is certainly reduced to zero at the blocking stage if the leading site is occupied by a vehicle, it is impossible to have the configurations $+1,0$ and $+1,+1$ immediately after the randomization stage; therefore,

$$
p_{2}(+1,0)=0
$$

and

$$
p_{2}(+1,+1)=0 .
$$

In the special case $p_{s}=0$ the 2 -cluster-like probabilities (A.1)-(A.9) reduce to the corresponding expressions for the true 2-cluster probabilities derived earlier [17] for the NaSch model. 


\section{B The 2-cluster equations}

Using the symbols $\mu=p_{s}+p q_{s}$ and $\nu=q q_{s}$ we can write the exact equations for time evolution of the 2-cluster probabilities $C_{2}\left(s_{1}, s_{2}\right)$ in the BJH model in terms of the 2-cluster and higher-cluster probabilities in the steady state as follows.

$$
\begin{aligned}
C_{2}(-1,-1) & =C_{3}(-1,-1,-1)+p C_{3}(0,-1,-1)+p C_{3}(1,-1,-1)+\mu C_{3}(2,-1,-1) \\
& +q C_{4}(-1,-1,0,-1)+p q C_{4}(0,-1,0,-1)+\mu q C_{4}(2,-1,0,-1) \\
& +p q C_{4}(1,-1,0,-1)+\nu C_{4}(-1,-1,2,-1)+p \nu C_{4}(0,-1,2,-1) \\
& +\mu \nu C_{4}(2,-1,2,-1)+p \nu C_{4}(1,-1,2,-1)+q C_{4}(-1,-1,1,-1) \\
& +p q C_{4}(0,-1,1,-1)+\mu q C_{4}(2,-1,1,-1)+p q C_{4}(1,-1,1,-1) \\
C_{2}(0,-1) & =p C_{2}(0,-1)+\mu C_{2}(2,-1)+p C_{2}(1,-1) \\
C_{2}(2,-1) & =q C_{2}(0,-1)+\nu C_{2}(2,-1)-q C_{3}(-1,0,-1)-\nu C_{3}(-1,2,-1) \\
& +q C_{3}(0,0,-1)+\nu C_{3}(0,2,-1)+q C_{3}(0,1,-1)+q C_{3}(2,1,-1) \\
& +q C_{3}(1,1,-1)-q C_{3}(0,0,-1)-\nu C_{3}(0,2,-1) \\
C_{2}(1,-1) & =q C_{3}(0,-1,-1)+q C_{3}(1,-1,-1)+\nu C_{3}(2,-1,-1)+q^{2} C_{4}(0,-1,0,-1) \\
& +q \nu C_{4}(2,-1,0,-1)+q^{2} C_{4}(1,-1,0,-1)+q \nu C_{4}(0,-1,2,-1) \\
& +\nu^{2} C_{4}(2,-1,2,-1)+q \nu C_{4}(1,-1,2,-1)+q^{2} C_{4}(0,-1,1,-1) \\
& +q \nu C_{4}(2,-1,1,-1)+q^{2} C_{4}(1,-1,1,-1) \\
& =p C_{4}(-1,-1,0,-1)+p^{2} C_{4}(0,-1,0,-1)+\mu p C_{4}(2,-1,0,-1) \\
& +p^{2} C_{4}(1,-1,0,-1)+\mu C_{4}(-1,-1,2,-1)+p \mu C_{4}(0,-1,2,-1) \\
& +\mu^{2} C_{4}(2,-1,2,-1)+p \mu C_{4}(1,-1,2,-1)+p C_{4}(-1,-1,1,-1) \\
& +p^{2} C_{4}(0,-1,1,-1)+p \mu C_{4}(2,-1,1,-1)+p^{2} C_{4}(1,-1,1,-1) \\
& \\
&
\end{aligned}
$$




$$
\begin{aligned}
C_{2}(0,2) & =0 \\
C_{2}(2,2) & =C_{2}(2,0)+C_{2}(1,0)+C_{2}(2,2)+C_{2}(1,2)-C_{3}(2,0,-1) \\
& -C_{3}(1,0,-1)-C_{3}(2,2,-1)-C_{3}(1,2,-1)+C_{2}(0,0) \\
& -C_{3}(0,0,-1)+C_{2}(0,2)-C_{3}(0,2,-1)+C_{2}(0,1)-C_{3}(0,1,-1) \\
& +C_{2}(2,1)-C_{3}(2,1,-1)+C_{2}(1,1)-C_{3}(1,1,-1) \\
C_{2}(1,2) & =q C_{3}(0,-1,0)-q C_{4}(0,-1,0,-1)+\nu C_{3}(2,-1,0)-\nu C_{4}(2,-1,0,-1) \\
& +q C_{3}(1,-1,0)-q C_{4}(1,-1,0,-1)+q C_{3}(0,-1,2)-q C_{4}(1,-1,2,-1) \\
& +\nu C_{3}(2,-1,2)-\nu C_{4}(2,-1,2,-1)+q C_{3}(1,-1,2)-q C_{4}(1,-1,2,-1) \\
& +q C_{3}(0,-1,1)-q C_{4}(0,-1,1,-1)+\nu C_{3}(2,-1,1)-\nu C_{4}(2,-1,1,-1) \\
& +q C_{3}(1,-1,1)-q C_{4}(1,-1,1,-1) \\
C_{2}(-1,1) & =q C_{2}(0,-1)+\nu C_{2}(2,-1)+q C_{2}(1,-1) \\
C_{2}(0,1) & =0 \\
C_{2}(2,1) & =0 \\
C_{2}(1,1) & =0 .
\end{aligned}
$$

In order to close these equations, we now make the approximate decomposition of the 3 -cluster and 4-cluster probabilities in terms of 2-cluster probabilties using the formulae

$$
\begin{gathered}
C_{3}\left(x_{1}, x_{2}, x_{3}\right)=\frac{C_{2}\left(x_{1}, x_{2}\right) C_{2}\left(x_{2}, x_{3}\right)}{\sum_{s=-1}^{2} C_{2}\left(x_{2}, s\right)} \\
C_{4}\left(x_{1}, x_{2}, x_{3}, x_{4}\right)=\frac{C_{2}\left(x_{1}, x_{2}\right) C_{2}\left(x_{2}, x_{3}\right) C_{2}\left(x_{3}, x_{4}\right)}{\left[\sum_{s=-1}^{2} C_{2}\left(s, x_{2}\right)\right] \cdot\left[\sum_{s=-1}^{2} C_{2}\left(x_{3}, s\right)\right]}
\end{gathered}
$$

We like to remark that the so-called Kolmogorov consistency conditions 38]

$$
\sum_{s=-1}^{2} C_{2}(-1, s)=\sum_{s=-1}^{2} C_{2}(s,-1)=1-c
$$

and

$$
\sum_{s=-1}^{2}\left[C_{2}(0, s)+C_{2}(1, s)+C_{2}(2, s)\right]=\sum_{s=-1}^{2}\left[C_{2}(s, 0)+C_{2}(s, 1)+C_{2}(s, 2)\right]=c
$$

are useful also for the numerical solution of the equations (B.2-B.16).

\section{Relation between COMF and hybrid approaches}

In order to point out the equivalence of the hybrid and COMF approaches, we sketch here the derivation of the distribution of the jam sizes in the BJH model with $V_{\max }=1$ following the hybrid approach and show that we get the same expression as we derived in section 4 following the pure COMF approach.

Decomposing the distribution $\mathcal{P}_{j s}(k)$ into the 2-cluster-like conditional probabilities $p_{2}(\underline{\mathrm{a}} \mid b)$ we get

$$
\mathcal{P}_{j s}(k)=\mathcal{N}_{j s} p_{2}(\underline{-1} \mid 0)\left[p_{2}(\underline{0} \mid 0)\right]^{k-1} p_{2}(\underline{0} \mid-1)+p_{2}(\underline{-1} \mid 0)\left[p_{2}(\underline{0} \mid 0)\right]^{k-1} p_{2}(\underline{0} \mid 1)
$$

where $\mathcal{N}_{j s}$ is a normalization coefficient. Using the expressions for the 2-cluster-like probabilities derived in the appendix $\mathrm{A}$ and normalizing the distribution to unity we recover [35] the expression (12) of $\mathcal{P}_{j s}(k)$ in terms of $\mathcal{P}(0)$. 


\section{References}

[1] M.J. Lighthill and G.B. Whitham, Proc. Roy. Soc. A 229, 281 (1955)

[2] B.S. Kerner, S.L. Klenov and P. Konhäuser, Phys. Rev. E 56, 4200 (1997)

[3] M.Y. Choi and H.Y. Lee, Phys. Rev. E 52, 5979 (1995)

[4] I. Prigogine and R. Herman, Kinetic Theory of Vehicular Traffic (Elsevier, Amsterdam, 1971)

[5] D. Helbing, Physica A 219, 375 (1995); 219, 391 (1995); 233, 253 (1995)

[6] T. Nagatani, J. Phys. Soc. Jap. 66, 1219 (1997)

[7] see, for reviews, R. Herman and K. Gardels, Sci. Amer. 209(6), 35 (1963);

D.C. Gazis, Science, 157, 273 (1967)

[8] M. Bando, K. Hasebe, A. Nakayama, A. Shibata and Y. Sugiyama, Phys. Rev. E 51, 1035 (1995)

[9] S. Yukawa and M. Kikuchi, J. Phys. Soc. Jap. 64, 35 (1995); 65, $916(1996)$

[10] T. Nagatani, J. Phys. Soc. Jap. 65, 2333 (1996).

[11] K. Nagel and M. Schreckenberg, J. Physique I, 2, 2221 (1992)

[12] K. Nagel and H.J. Herrmann, Physica A 199, 254 (1993)

[13] M. Schreckenberg, A. Schadschneider, K. Nagel and N. Ito, Phys. Rev. E, 51, 2939 (1995); also A. Schadschneider and M. Schreckenberg, J. Phys. A 26, L679 (1993)

[14] A. Schadschneider and M. Schreckenberg, J. Phys. A 30, L69 (1997).

[15] A. Schadschneider and M. Schreckenberg, J. Phys. A 31, L225 (1998)

[16] K. Nagel, Phys. Rev. E 53, 4655 (1996)

[17] D. Chowdhury, A. Majumdar, K. Ghosh, S. Sinha and R.B. Stinchcombe, Physica A 246, 471 (1997)

[18] K. Ghosh, A. Majumdar and D. Chowdhury, Phys. Rev. E 58, 4012 (1998)

[19] D. Chowdhury, A. Pasupathy and S. Sinha, Euro. Phys. J. B 5, 781 (1998)

[20] A. Schadschneider, in: Traffic and Granular Flow'97, M. Schreckenberg and D.E. Wolf (eds.) (Springer, Singapore, 1998)

[21] D. Ktitarev, D. Chowdhury and D.E. Wolf, J. Phys. A 30, L221 (1997)

[22] Z. Csahok and T. Vicsek, J. Phys. A 27, L591 (1994)

[23] K. Nagel and M. Paczuski, Phys. Rev. E 51, 2909 (1995)

[24] N. Boccara, H. Fuks and Q. Zeng, J. Phys. A 30, 3329 (1997)

[25] S.C. Benjamin, N.F. Johnson and P.MḦui, J. Phys. A 29, 3119 (1996)

[26] M. Takayasu and H. Takayasu, Fractals 1, 860 (1993) 
[27] R. Barlovic, L. Santen, A. Schadschneider and M. Schreckenberg, Eur. Phys. J. 5, $793(1998)$

[28] A. Schadschneider and M. Schreckenberg, Ann. Physik 6, 541 (1997)

[29] D.E. Wolf, Physica A (in press)

[30] B. Schmittmann and R.K.P. Zia in: Phase Transitions and Critical Phenomena, vol.17, eds. C. Domb and J.L. Lebowitz (Academic Press, 1995)

[31] A.D. May, Traffic Flow Fundamentals (Prentice-Hall, 1990)

[32] D.E. Wolf, M. Schreckenberg and A. Bachem (eds.) Traffic and Granular Flow (World Scientific, 1996).

[33] M. Schreckenberg and D.E. Wolf (eds.) Traffic and Granular Flow'97 (Springer, Singapore, 1998);

[34] S. Wolfram, Theory and Applications of Cellular Automata (World Scientific, 1986)

[35] S. Sinha, Masters (Theory) Thesis, IIT Kanpur (1998) (unpublished)

[36] B.S. Kerner and H. Rehborn, Phys. Rev. Lett. 79, 4030 (1997)

[37] B.S. Kerner and H. Rehborn, Phys. Rev. E 53, R1297 (1996)

[38] H.A. Gutowitz, D. Victor and B.W. Knight, Physica 28D, 18 (1987) 